\title{
Serum MicroRNA-155 Expression Level as a Novel Biomarker in Alopecia Areata Patients \\ N.E.Sorour ${ }^{1}$, A.H.Hamed ${ }^{1}$, H.A.Tabl ${ }^{2}$ and SH.G.Mousa ${ }^{2}$ \\ ${ }^{1}$ Dermatology, Venereology and Andrology Dept., Faculty of Medicine, Benha Univ, Benha, Egypt \\ ${ }^{2}$ Medical Microbiology and Immunology Dept.,Faculty of Medicine, Benha Univ, Benha,Egypt \\ E-Mail:Dr.ShimmaaGamal@gmail.com
}

\section{Abstract}

MicroRNA-155 has been implicated in several autoimmune diseases, including experimental autoimmune encephalomyelitis and rheumatoid arthritis, due to its activity against a wide range of immunological gene targets. Overexpression of miR-155 may represent a hallmark feature in Alopecia areata (AA) disease pathogenesis and across multiple autoimmune conditions. It could potentially reflect and or influence the activation of $\mathrm{T}$ cells in autoimmune diseas. The present work aims is to assess serum level of miRNA-155 in patients with AA. MiR-155 level was identified by real-time polymerase chain reaction (PCR) in 30 patients with AA and 30 individuals as healthy controls. Results: There was a statistically significant increase in serum miR-155 level in patients than in controls. Conclusion: Serum level of miR-155 was significantly higher in patients with AA than controls, which indicated its role in the pathogenesis of AA.

Keywords: Alopecia Areata, polymerase chain reaction , MicroRNA.

\section{Introduction}

Alopecia areata is an autoimmune disease that is characterized by acute onset of non-scarring hair loss as usually sharply defined areas. Any hair-bearing area can be affected, but the most noticeable are the scalp, the beard area and the eyebrows [2]. The etiology of AA appears to be multifactorial. There is strong evidence of a genetic basis for AA; the predisposition has been shown to be polygenic [3]. Evidence has suggested the role of altered T-cell-mediated immunity and cytokines in the pathogenesis of AA. Th1 cells predominantly produce interferon gamaa (IFN- $\gamma$ ), tumour necrosis factor beta (TNF- $\beta$ ) and interleukin (IL-2); Th2 cells produce IL-4, IL-5, IL-10 and IL-13; Th17 cells produce IL-17, IL-23 and IL-6; and Tregs synthesize transforming growth factor beta (TGF- $\beta$ ) and IL-10[4].

Differential levels of circulating miRNAs have been identified in autoimmune diseases sharing similar pathogenic pathways to AA, such as rheumatoid arthritis and type I diabetes mellitus [5]. MicroRNA155 has been implicated in several autoimmune diseases, including experimental autoimmune encephalomyelitis and rheumatoid arthritis, due to its activity against a wide range of immunological gene targets. Overexpression of miR-155 may represent a hallmark feature in AA disease pathogenesis and across multiple autoimmune conditions. It could potentially reflect and or influence the activation of $\mathrm{T}$ cells in autoimmune diseases [1]. Dysregulation of T-cell costimulatory signals is one of the most important pathways associated with autoimmunity. MiRNA causes dysregulation of CTLA4 (Cytotoxic Tlymphocyte-associated protein 4) which considered an inhibitor of T-cell activity, and ICOS (Inducible T-cell co-stimulator), has been identified in rheumatoid arthritis in humans [6].

\section{Material and methods}

This study has been conducted on 30 patients clinically diagnosed as AA (group A) selected from the Outpatient Clinic of the Dermatology and Andrology Department of Benha University Hospitals. In addition, 30 apparently healthy individuals of matched .

Medicine. A signed written informed consent was obtained from each patient to participate in the study. All studied subjects were tested for serum level miR155.Two ml venous blood was collected from each subject by clean venipuncture using disposable plastic syringe and placed on plain tube (without age and sex were chosen as a control group (group B).

The protocol was submitted and approved by the Ethics Committee on research involving human subjects of Benha Faculty of anticoagulant) for serum separation. The tube was left at room temperature for 30 minutes till coagulation, and then was centrifuged (at $1500 \mathrm{rpm}$ for 15 minutes). The resultant serum was stored at $-20^{\circ} \mathrm{C}$ for further testing. Using the microRNA extraction kit "miRNeasy Mini Kit” (Qiagen, Germany), RNA was isolated from serum according to manufacturer's instruction .

\section{Results and discussion}

Alopecia areata is considered as an autoimmune disorder which CD4+ and CD8+ T-cells violate the immune privilege of the anagen hair follicle, leading to loss of the growing hair shaft, CD8+ T-cells are present in significantly greater quantities than $\mathrm{CD} 4+$ cells, and a subset of them known as CD8+ NKG2D+ T-cells has been found both necessary and sufficient to induce AA lesion. A predominant Th1 cytokine profile has been discovered at the site of AA lesions [7]. MicroRNA-155 has been implicated in several autoimmune diseases, including experimental autoimmune encephalomyelitis and rheumatoid arthritis, due to its activity against a wide range of immunological gene targets. It could potentially reflect and or influence the activation of $\mathrm{T}$ cells in autoimmune diseases [1].

In the present study, has been investigated in patients with AA and healthy control subjects of Egyptian origin. 
The aim of this study is to assess serum level of miRNA155 in patients with AA.

This present study included 30 patients suffering from AA. In addition to 30 apparently healthy individuals of matched age and sex as a control group. Patients were recurrited from the outpatient clinic of Dermatology and Andrology Department of Benha University Hospitals. The study was approved by the local ethic committee and an informed consent was obtained from each individual being enrolled in the study.

The results of the present study revealed that serum levels of miR-155 were significantly higher in AA patients Table(1) and Fig (1). The role of miR-155 in pathogenesis of AA could be caused by dysregulation of CTLA-4 which considered an inhibitor of T-cell activity, the later has been identified in autoimmune diseases e.g; RA in humans [6].

Table (1) Comparison of serum level of miR-155 between patients and control group:

\begin{tabular}{|c|c|c|c|c|c|c|c|c|}
\hline \multirow[t]{2}{*}{ Variable } & \multicolumn{3}{|c|}{ Patients $(\mathrm{N}=30)$} & \multicolumn{3}{|c|}{ Controls $(\mathrm{N}=30)$} & \multirow[t]{2}{*}{$\mathrm{Z}_{\mathrm{MWU}}$ test } & \multirow[t]{2}{*}{$\mathbf{P}$} \\
\hline & Mean \pm SD & Range & Median (IQ & Mean \pm SD & Range & Median(IQR) & & \\
\hline Serum miR-155 & $5.2 \pm 7.3$ & $0.03-19.34$ & 1.54 & $1.76 \pm 1.2$ & $0.02-3.20$ & 0.43 & 0.52 & $<0.05$ \\
\hline
\end{tabular}

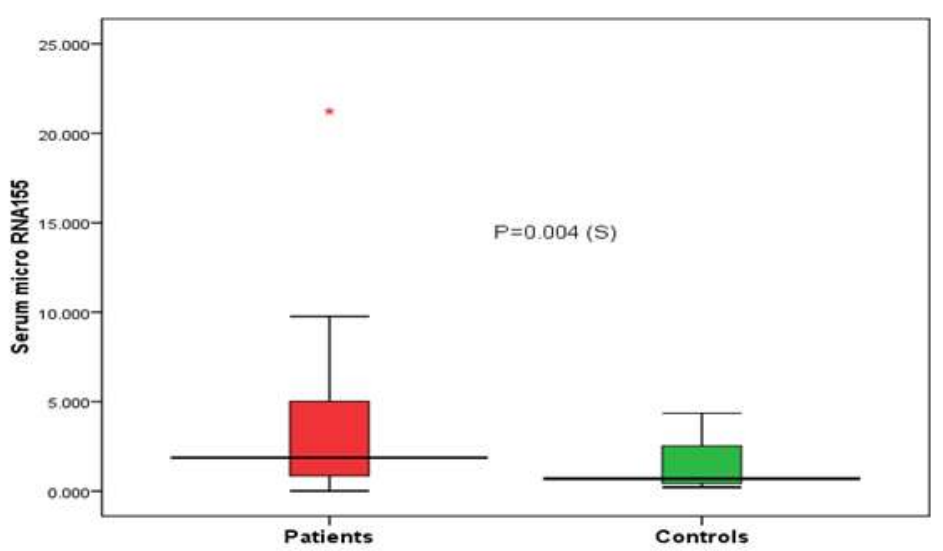

Fig (1) Box plot showing the median and inter quartile range (IQR) of serum miR-155 in patients and controls.

Another explanation for the role of miR-155 in pathogenesis of AA is through miR-155's positive role in Th17 cell development. On one hand, miR-155 might function to block the inhibitory impact of cytokines such as IL-4 and IFN- $\gamma$ on the Th17 cell differentiation pathway. Micro RNA-155 has been shown to limit production of IL-4 by CD4+ $\mathrm{T}$ cells through repression of the transcription factor c-Maf [7].

\section{Conclusion}

From the results of present study, we can conclude that serum level of miR-155 was significantly higher in patients with AA than controls, which indicated its role in the pathogenesis of AA.

\section{References}

[1] R.M. O'Connell , D. Kahn , W.S. Gibson , J.L. Round , R.L .Scholz , A.A. Chaudhuri MicroRNA155 promotes autoimmune inflammation b y enhancing inflammatory $\mathrm{T}$ cel development. Immunity. ; Vol.33(4)PP.607-619, 2010.
[2] C. Pratt, L.King , A. Messenger , A. Christiano Alopecia areata. Nat Rev Dis Primers ;Vol.3 (3),PP.17011, 2017.

[3] R. Dudda -Subramanya , A .Alexis , K.. Siu , A. Sinha ,Alopecia areata: genetic complexity underlies clinical heterogeneity. Eur J Dermatol. Vol.17(5),PP.367-374, 2007.

[4] A. O'Garra and p .Vieira ,Regulatory $\mathrm{T}$ cells and mechanisms of immune system control. Nat Med. Vol.10(8),PP.801-805, 2004.

[5] A.D. McClelland and P. Kantharidis ,microRNA in the development of diabetic complications.Clin Sci (Lond). Vol.126(2),PP.95-110, 2014.

[6] X. Luo , L.M. Tsai , N. Shen , D. Yu ,Evidence for microRNA-mediated regulation in rheumatic diseases. Ann Rheum Dis. Vol.69 ,PP. 30-36,1973, 2010.

[7] A. Rodriguez , E. Vigorito , S. Clare , M.V. Warren , P. Couttet , D.R. Soond ,Requirement of bic/microRNA-155 for normal immune function. Science. Vol.316(5824), PP.608-611,2007. 